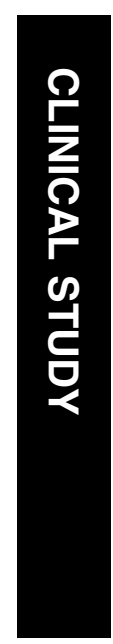

\section{Risk factors for cystoid macular oedema in patients with uveitis}

B van Kooij ${ }^{1}$, K Probst $^{1}$, R Fijnheer ${ }^{2}$, M Roest ${ }^{2}$, W de Loos $^{3}$ and A Rothova'
${ }^{1}$ Department of

Ophthalmology, FC Donders Institute of Ophthalmology, University Medical Center, Utrecht, The Netherlands

${ }^{2}$ Department of Haematology, University Medical Center, Utrecht, The Netherlands

${ }^{3}$ Department of Internal Medicine, University Medical Center, Utrecht, The Netherlands

Correspondence:

B van Kooij,

Department of

Ophthalmology,

FC Donders Institute of Ophthalmology,

University Medical Center Utrecht, PO Box 85 500,

Utrecht 3508 GA,

The Netherlands

Tel: + 3130 2507880;

Fax: + 31302505417 .

E-mail: b.vankooij@

oogh.azu.nl

$B$ van Kooij and $K$ Probst contributed equally to this work

Received: 2 March 2006 Accepted in revised form: 8 August 2006

Published online: 6 October 2007

\begin{abstract}
Purpose To determine ophthalmologic and systemic factors associated with the presence of cystoid macular oedema (CMO) in patients with uveitis.
\end{abstract}

Methods Retrospective cross-sectional study in which 97 consecutive patients with uveitis filled in an extensive questionnaire for the presence of cardiovascular diseases and its risk factors. An analysis of the ophthalmologic and questionnaire data was conducted.

Results CMO was present in $44 \%$ (43/97) of patients. Its presence was strongly associated with increasing age $(P=0.001)$ and age at onset of uveitis $(P<0.001)$. For patients older than 50 years, the risk of having CMO was 3.8-fold (95\% confidence intervals 1.6-9.0) larger than for younger patients. The most frequent anatomic location of uveitis associated with CMO was panuveitis (49\%). Papillary leakage on fluorescein angiography was associated with CMO $(P<0.001)$, independently of other risk factors. After adjustment for age, multivariate logistic regression showed no association between cardiovascular disease and its risk factors and the presence of CMO. Conclusions Age, independent of duration of uveitis, was a major risk factor for the presence of CMO in uveitis. A positive correlation between $\mathrm{CMO}$ and papillary leakage on angiography was noted. Eye (2008) 22, 256-260; doi:10.1038/sj.eye.6702595; published online 6 October 2007

Keywords: cystoid macular oedema; uveitis; cardiovascular disease; age

\section{Introduction}

Cystoid macular oedema (CMO) is a major cause of visual loss in patients with uveitis. ${ }^{1,2}$ Despite aggressive treatments, progression of $\mathrm{CMO}$ with accompanying visual loss is common. ${ }^{3}$ The pathogenesis of inflammatory $\mathrm{CMO}$ is largely unknown, as are the factors that determine either visual recovery or irreversible loss of vision in an individual patient with CMO. Several studies have pointed out the increased frequency of systemic cardiovascular disorders (CVDs) in CMO due to diabetic retinopathy, central retinal vein occlusion, and post intraocular surgery. ${ }^{4-8}$ Recent reports showed that systemic microvascular damage, which is a risk factor for developing CVD, was associated with inflammatory and diabetic CMO. ${ }^{9,10}$ Herein, we evaluate the impact of age, presence of CVD, CVD risk factors, and ophthalmologic data in uveitis patients with and without CMO.

\section{Materials and methods}

This study included 100 consecutive patients with uveitis who consulted our uveitis clinic that combines secondary and tertiary ophthalmologic care. All patients were checked for a history of cardiovascular diseases or their risk factors using an extensive questionnaire used in the department of vascular medicine. ${ }^{11}$ Three uveitis patients were left out of the analysis, because of incomplete data sets. This study was performed according to the tenets of the Declaration of Helsinki.

The variables collected were the general medical history, medication, age, age at onset of uveitis, duration of uveitis and CMO, family history of CVDs, and presence of CVD and its risk factors. The diagnosis of cardiovascular disease included a history of myocardial infarction, angina pectoris, coronary arterial bypass graft, aortic aneurysm, ischaemic stroke, transient ischaemic attacks, carotid artery occlusion, and intermittent claudication. Risk factors for CVD in this study were hypertension, hypercholesterolaemia, smoking, pack-years, and higher BMI. ${ }^{11}$ Pack-years were calculated by multiplying the duration of 
smoking by the number of cigarettes smoked per day, divided by $20 .{ }^{11}$ Patients with diabetes mellitus were excluded from this study; therefore, this risk factor was not included in our calculations.

In addition, we conducted a retrospective analysis of the ophthalmologic data of all patients with uveitis, with the emphasis on specific diagnosis and location of uveitis, presence, onset, and severity of CMO. ${ }^{12}$ The presence of $\mathrm{CMO}$, vasculitis, papillary leakage, retinal occlusions, or multiple retinal haemorrhages were evaluated on fluorescein angiograms. The fluorescein angiograms $(n=83)$ were evaluated by the researchers (KP) and by an experienced, masked ophthalmologist (AR). In the remaining 14 patients were the above symptoms and CMO defined by clinical criteria. ${ }^{13,14}$

For statistical analysis $\left(\chi^{2}\right.$, Kruskall-Wallis, MannWhitney $U$-tests for nonparametric analysis, univariate, and multivariate logistic regression), SPSS version 12.0 was used.

\section{Results}

Ophthalmologic and cardiovascular data of our uveitis patients are given in Table 1 . The mean age at participation in our study was 47.5 years (range 12.3 82.5; median 45.3) and the mean follow-up (date of onset of uveitis until the date of completing the questionnaire) 8.4 years (range $0.0-40.9$, median 6.6). The male-tofemale ratio was $1: 1.9(34 / 63)$.

Overall analysis of our group of 97 uveitis patients showed CMO to be present in $43 / 97(44 \%)$, vasculitis in $41 / 85(48 \%)$, papillary leakage in $41 / 77(53 \%)$, and retinal occlusions in 10/78 (13\%; Table 1$)$.

In patients with $\mathrm{CMO}$, panuveitis was the most frequent anatomic location of uveitis $(49 \%, P=0.001)$. Uveitis patients with $\mathrm{CMO}$ also exhibited papillary leakage on fluorescein angiography $(25 / 38,66 \%$ vs $16 / 39$, $41 \%, P=0.03)$ more often. The presence of $\mathrm{CMO}$ was strongly associated with a higher age of our patients (54.7 vs 41.8 years, $P<0.001)$. Uveitis patients with $\mathrm{CMO}$ were older at the onset of uveitis $(47.1$ vs $32.9, P<0.001)$ and, in univariate analysis, showed higher BMI (25.0 vs 23.6, $P=0.045)$ and more pack-years (14.2 vs 5.1 years, $P=0.002$; Table 1). The duration of uveitis was not associated with the presence of $\mathrm{CMO}(P=0.4)$. The prevalence of CVD among patients with or without $\mathrm{CMO}$ did not differ significantly $(7 / 43,16 \%$ vs $3 / 54,6 \%$, $P=0.1$ ).

Table 1 General and ophthalmological characteristics of uveitis patients, with and without CMO

\begin{tabular}{|c|c|c|c|c|}
\hline & Total & $\mathrm{CMO}+$ & $\mathrm{CMO}-$ & P-value ${ }^{\mathrm{a}}$ \\
\hline Number of patients & 97 & $43(44 \%)$ & $54(56 \%)$ & - \\
\hline Number of eyes & 171 & $79(46 \%)$ & $92(54 \%)$ & - \\
\hline Male/female & $1: 1.9$ & $1: 1.5$ & $1: 2.2$ & NS \\
\hline Mean age (years) & 47.5 & 54.7 & 41.8 & $<0.001$ \\
\hline Mean age at onset of uveitis (years) & 39.2 & 47.1 & 32.9 & $<0.001$ \\
\hline Mean duration of uveitis (years) & 8.4 & 7.6 & 9.0 & NS \\
\hline Uni-/bilateral disease & $23 / 74$ & $8 / 35$ & $15 / 39$ & NS \\
\hline \multicolumn{5}{|l|}{ Location of uveitis } \\
\hline Anterior & $7(7 \%)$ & $0(0 \%)$ & $7(13 \%)$ & 0.01 \\
\hline Intermediate & $17(19 \%)$ & $8(19 \%)$ & $9(17 \%)$ & NS \\
\hline Posterior & $43(44 \%)$ & $14(33 \%)$ & $29(54 \%)$ & 0.02 \\
\hline Panuveitis & $30(31 \%)$ & $21(49 \%)$ & $9(17 \%)$ & 0.001 \\
\hline \multicolumn{5}{|l|}{ Retinal changes ${ }^{\mathrm{b}}$} \\
\hline Vasculitis & $41 / 85(48 \%)$ & $24 / 41(59 \%)$ & $17 / 44(39 \%)$ & NS \\
\hline Papillary leakage & $41 / 77(53 \%)$ & $25 / 38(66 \%)$ & $16 / 39(41 \%)$ & 0.03 \\
\hline Retinal branch occlusions & $10 / 78(13 \%)$ & $7 / 38(18 \%)$ & $3 / 40(8 \%)$ & NS \\
\hline Retinal haemorrhages & $19 / 80(24 \%)$ & $14 / 39(36 \%)$ & $5 / 41(12 \%)$ & NS \\
\hline \multicolumn{5}{|l|}{ Cardiovascular data } \\
\hline Cardiovascular disease & $10(10 \%)$ & $7(16 \%)$ & $3(6 \%)$ & NS \\
\hline Hypertension & $13(13 \%)$ & $8(19 \%)$ & $5(9 \%)$ & NS \\
\hline Hypercholesterolaemia & $12(12 \%)$ & $6(14 \%)$ & $6(11 \%)$ & NS \\
\hline Smoking & $60(62 \%)$ & $31(72 \%)$ & $29(54 \%)$ & NS \\
\hline Mean pack-years & 9.2 & 14.2 & 5.1 & 0.002 \\
\hline Mean body mass index & 24.2 & 25.0 & 23.6 & 0.045 \\
\hline
\end{tabular}

NS = nonsignificant.

aUnivariate analysis of $\mathrm{CMO}+$ vs $\mathrm{CMO}-$ uveitis patients

${ }^{\mathrm{b}}$ The numbers indicate the patients in which the specific retinal changes could be clinically examined and/or sufficiently evaluated on fluorescein angiograms. 
Table 2 Multivariate logistic regression analysis to obtain independent factors associated with cystoid macular oedema ${ }^{a}$

\begin{tabular}{lcc}
\hline & Significance & Odds ratio (95\% CI) \\
\hline Age at onset (years) & $<0.01$ & $1.09(1.0-1.1)$ \\
Papillary leakage & 0.01 & $6.06(1.4-25.6)$ \\
Retinal occlusions & 0.09 & $9.9(0.7-141.7)$ \\
\hline
\end{tabular}

CI, confidence interval.

aStepwise backward binary logistic regression

Multivariate logistic regressions showed that age at onset and papillary leakage were the most important independent factors associated with CMO (Table 2). There was a trend indicating retinal occlusions as an independent risk factor for the presence of $\mathrm{CMO}$ (odds ratio 9.9; 95\% confidence interval (CI) 0.7-141.7; Table 2), but due to the lack of statistical power this finding did not reach significance. The relationship between BMI, pack-years, other CVD factors, location of uveitis (panuveitis), and CMO diminished after adjustment for age.

Uveitis patients older than 50 years $(n=41)$, had a 3.8fold higher risk of having $\mathrm{CMO}$ than patients younger than $50(n=56 ; 95 \%$ CI 1.6-9.0), and patients above 70 $(n=12)$ had even a 4.5 -fold higher risk (95\% CI 1.2-19.6). In patients above 50 who also exhibited papillary leakage, the prevalence of CMO was $81 \%(13 / 16)$. Patients younger than 50 with concomitant papillary leakage showed CMO in $48 \%(12 / 25)$, whereas in younger patients without papillary leakage, the prevalence of CMO was only $11 \%(2 / 19, P=0.008)$. CMO in patients above 50 with panuveitis was noted in $11 / 13$ $(85 \%)$ of patients in contrast to $14 / 28(50 \%)$ in patients without panuveitis $(P=0.03)$.

\section{Discussion}

We observed a strong association between advancing age and presence of $\mathrm{CMO}$ in uveitis patients. The risk of the presence of $\mathrm{CMO}$ in patients older than 50 years was 3.8-fold higher compared to those younger than 50 years. This association was independent of the duration of uveitis. In addition, papillary leakage was an independent factor associated with the presence of $\mathrm{CMO}$. CMO in uveitis was also associated with increased BMI, prolonged heavy smoking, and location of uveitis (panuveitis), but these associations diminished after adjustment for age.

Determinants of early development of CMO have not been systematically investigated in the past. Incidental reports showed several characteristics that preceded the development of CMO in uveitis, including decreased contrast sensitivity, abnormal colour vision, and photoreceptor dysfunction and/or loss. ${ }^{15,16}$ The prevalence of CMO has further been associated with specific uveitis entities including pars planitis, Behçet's syndrome, birdshot chorioretinopathy, and peripheral multifocal choroiditis. ${ }^{17-19}$ In this present study, the most important prognosticator for the presence of inflammatory CMO was the advancing age of the patient, which seemed to be independent of the duration of uveitis. A longitudinal prospective study should be performed to see if these factors contributed to the development of CMO.

An association between the prolonged duration of uveitis and the presence of CMO has been noted previously by others, ${ }^{12,20}$ however, the effect of the absolute age of the uveitis patients on $\mathrm{CMO}$ was not investigated. Hypothetical explanations for this finding might include decreased function and/or degeneration of retinal cells during life, a process that may be enhanced by an intraocular inflammation. ${ }^{21}$ The eventual compromised condition of the vascular wall in aged persons, associated with increasing leakage of fluid through the vessels combined with a reduced ability of reabsorption of fluid through the elderly retinal pigment epithelium and Bruch's membrane, might also play a role. In the literature, the negative influence of age on the visual outcome of CMO has been described in retinal occlusions and diabetic retinopathy, and was attributed to insufficiency of the vascular endothelium with a subsequent increase of leakage of fluids, or inability to reabsorb fluid in older patients. ${ }^{22-25}$

Surprisingly, no association between the duration of the uveitis and CMO was noted in our study. This contradiction with previous reports can be explained by the large number of patients with chronic uveitis included in our series (the mean duration of uveitis in our $\mathrm{CMO}$ group was 7.6 years and in our non-CMO group 9.0 years). It is probable that the duration of uveitis might influence the development of CMO, especially during the first months or years after the onset of the intraocular inflammation. The influence of duration of the inflammatory process on the development of CMO probably decreases in cases that had already become chronic (as in the majority of our patients).

In our patients, we did not observe a significant correlation between CVD and/or its risk factors, except for age and the presence of CMO. An objective method to measure generalized vascular disease would require microalbuminuria and/or intima-media thickness measurements, which was beyond the scope of this study. In a recent study, however, we reported that trace microalbuminuria was noted in patients with uveitis and $\mathrm{CMO}$ in contrast to uveitis patients without CMO. This indicates that systemic microvascular leakage is 
associated with the development of CMO in uveitis patients. ${ }^{10}$ Therefore, we cannot entirely exclude the association between CVD and/or its risk factors and inflammatory $\mathrm{CMO}$, also because our study included only a limited number of patients with CVD. To analyse the actual impact of CVD and its risk on the development and prognosis of $\mathrm{CMO}$, a large age and gender-matched study of preferably elderly uveitis patients would be required.

The use of a questionnaire to assess the presence of CVD is a frequently employed and fairly reliable tool, but remains a subjective method, relying on the patients' cooperation and memory. ${ }^{26}$ We used fluorescein angiography to analyse the inflammatory $\mathrm{CMO}$. In a condition of chronic $\mathrm{CMO}$, one may be looking for subtle changes that can be picked up only on optical coherence tomography (OCT), which is a more sensitive technique for evaluating macular thickness. Unfortunately, OCT was not available for our study. Another possible drawback of this retrospective study from a tertiary centre is an element of selection bias (patients with worse disease tend to be retained and those with mild disease lost to follow up). Further, additional referral bias is also present because the patients with mild disease would not be referred to our centre.

Unexpectedly, a firm association between papillary leakage and CMO was observed. The association of CMO and optic disc leakage has also been reported following cataract extraction (Irvine-Gass syndrome). The pathogenesis of Irvine-Gass syndrome is attributed to mechanical forces in the vitreous and attachments of the vitreous around the macula and optic disc, as well as to an inflammatory component. ${ }^{27,28}$ In phakic uveitis patients, the pathogenesis of concomitant optic disc leakage with $\mathrm{CMO}$ is unknown, but mechanical traction or adhesions of the vitreous might also play a role, considering the improvement of inflammatory $\mathrm{CMO}$ after pars plana vitrectomy. ${ }^{3,29}$ An analysis of the natural history of the development of CMO would be necessary to clarify whether or when inflammatory $\mathrm{CMO}$ is preceded or accompanied by leakage of the optic disc.

Our study points out that elderly patients or patients with onset of uveitis at advanced age and patients with optic disk leakage carry an increased risk of having CMO. Our observations indicate that an earlier and forceful treatment of uveitis in elderly patients might postpone the development of CMO and thereby improve the visual prognosis of elderly patients with uveitis.

\section{Acknowledgements}

This study was supported in part by the Dr FP Fischer Foundation, Utrecht, The Netherlands.

\section{References}

1 Rothova A, Suttorp-van Schulten MS, Treffers WF, Kijlstra A. Causes and frequency of blindness in patients with intraocular inflammatory disease. Br J Ophthalmol 1996; 80: 332-336.

2 Durrani OM, Tehrani NN, Marr JE, Moradi P, Stavrou P, Murray PI. Degree, duration, and causes of visual loss in uveitis. Br J Ophthalmol 2004; 88: 1159-1162.

3 Rothova A. Medical treatment of cystoid macular edema. Ocul Immunol Inflamm 2002; 10: 239-246.

4 Jain R, Stevens JD, Bunce CV, Garrett C, Hykin PG. Ischaemic heart disease may predispose to pseudophakic cystoid macular oedema. Eye 2001; 15: 34-38.

5 Jalkh AE, Trempe CL. Macular edema in branch retinal vein occlusion: types and treatment. Ophthalmic Surg 1989; 20: 26-32.

6 Lopes de Faria JM, Jalkh AE, Trempe CL, MCMOel JW. Diabetic macular edema: risk factors and concomitants. Acta Ophthalmol Scand 1999; 77: 170-175.

7 Wallow IH, Danis RP, Bindley C, Neider M. Cystoid macular degeneration in experimental branch retinal vein occlusion. Ophthalmology 1988; 95: 1371-1379.

8 Gilbert RE, Tsalamandris C, Allen TJ, Colville D, Jerums G. Early nephropathy predicts vision-threatening retinal disease in patients with type I diabetes mellitus. J Am Soc Nephrol 1998; 9: 85-89.

9 Knudsen ST, Bek T, Poulsen PL, Hove MN, Rehling M, Mogensen CE. Macular edema reflects generalized vascular hyperpermeability in type 2 diabetic patients with retinopathy. Diabetes Care 2002; 25: 2328-2334.

10 van Kooij B, Fijnheer R, Roest M, Rothova A. Trace microalbuminuria in inflammatory cystoid macular edema. Am J Ophthalmol 2004; 138: 1010-1015.

11 Simons PC, Algra A, Bots ML, Grobbee DE, van der GY Common carotid intima-media thickness and arterial stiffness: indicators of cardiovascular risk in high-risk patients. The SMART Study (second manifestations of arterial disease). Circulation 1999; 100: 951-957.

12 Lardenoye CW, van Schooneveld MJ, Treffers WF, Rothova A. Grid laser photocoagulation for macular oedema in uveitis or the Irvine-Gass syndrome. Br J Ophthalmol 1998; 82: 1013-1016.

13 Photocoagulation for diabetic macular edema. Early Treatment Diabetic Retinopathy Study report number 1. Early Treatment Diabetic Retinopathy Study research group. Arch Ophthalmol 1985; 103: 1796-1806.

14 Treatment techniques clinical guidelines for photocoagulation of diabetic macular edema. Early Treatment Diabetic Retinopathy Study Report Number 2. Early Treatment Diabetic Retinopathy Study Research Group. Ophthalmology 1987; 94: 761-774.

15 Ibanez HE, Lesher MP, Singerman LJ, Rice TA, Keep GF. Prospective evaluation of the effect of pseudophakic cystoid macula edema on contrast sensitivity. Arch Ophthalmol 1993; 111: $1635-1639$.

16 Lardenoye CW, Probst K, DeLint PJ, Rothova A. Photoreceptor function in eyes with macular edema. Invest Ophthalmol Vis Sci 2000; 41: 4048-4053.

17 Freeman G, Matos K, Pavesio CE. Cystoid macular oedema in uveitis: an unsolved problem. Eye 2001; 15: 12-17.

18 Lardenoye CW, Van der LA, de Loos WS, Treffers WF, Rothova A. Peripheral multifocal chorioretinitis: a distinct clinical entity? Ophthalmology 1997; 104: 1820-1826. 
19 Rothova A, Berendschot TT, Probst K, van KB, Baarsma GS Birdshot chorioretinopathy: long-term manifestations and visual prognosis. Ophthalmology 2004; 111: 954-959.

20 Dana MR, Merayo-Lloves J, Schaumberg DA, Foster CS. Prognosticators for visual outcome in sarcoid uveitis. Ophthalmology 1996; 103: 1846-1853.

21 Weiter JJ, Roh S, Pruett RC. Aging in the retina and choroid In: Albert DM, Jacobiec FA (eds). Principles and Practice of Ophthalmology. Basic Sciences. WB Saunders Company: Philadelphia, 1994, pp 718-722.

22 Bresnick GH. Diabetic macular edema. A review. Ophthalmology 1986; 93: 989-997.

23 Glacet-Bernard A, Coscas G, Chabanel A, Zourdani A, Lelong F, Samama MM. Prognostic factors for retinal vein occlusion: prospective study of 175 cases. Ophthalmology 1996; 103: 551-560.

24 Klein R, Klein BE, Moss SE, Davis MD, DeMets DL. The Wisconsin epidemiologic study of diabetic retinopathy.
IV. Diabetic macular edema. Ophthalmology 1984; 91: 1464-1474.

25 Moss SE, Klein R, Klein BE. The 14-year incidence of visual loss in a diabetic population. Ophthalmology 1998; 105: 998-1003.

26 Klungel OH, de Boer A, Paes AH, Seidell JC, Bakker A. Cardiovascular diseases and risk factors in a populationbased study in The Netherlands: agreement between questionnaire information and medical records. Neth $\mathrm{J} \mathrm{Med}$ 1990; 55: 177-183.

27 Blair NP, Kim SH. Cystoid macular edema after ocular surgery. In: Albert DM, Jacobiec FA (eds). Principles and Practice of Ophthalmology. Basic Sciences. WB Saunders Company: Philadelphia, 1994, pp 903-904.

28 Sebag J, Balazs EA. Pathogenesis of cystoid macular edema: an anatomic consideration of vitreoretinal adhesions. Surv Ophthalmol 1984; 28: 493-498.

29 Bovey EH, Herbort CP. Vitrectomy in the management of uveitis. Ocul Immunol Inflamm 2000; 8: 285-291. 